\title{
Plasma interferometry at high pressures
}

\author{
Kamran Akhtar, ${ }^{\text {a) }}$ John E. Scharer, Shane M. Tysk, and Enny Kho \\ Department of Electrical and Computer Engineering, University of Wisconsin, Madison, Wisconsin 53706
}

(Received 17 September 2002; accepted 1 November 2002)

A general formulation for the measurement of plasma density and effective collision frequency for lowly as well as highly, collisional plasmas using millimeter wave interferometry is presented. In the presence of high density and collisionality at high gas pressures where the collision frequency $(\nu)$ is of the order of both the plasma $\left(\omega_{p}\right)$ and the wave frequency of the millimeter wave $(\omega)$ ( $\nu$ $\left.\sim \omega, \omega_{p}\right)$, the measured line-average plasma density has a complex dependence on phase shift as well as the amplitude change of the millimeter wave signal. The measurement scheme and analysis presented in this article show that for collisional plasmas, simultaneous measurement of the phase change and the amplitude change data is required to uniquely determine the plasma density and collision frequency. The treatment allows the application of millimeter wave interferometry to a wide range of relative collision frequency, wave frequency and plasma frequency since it uniquely determines the line-average plasma density and effective collision frequency using the phase and amplitude change data. This diagnostic is particularly valuable at plasma densities $\left(n_{e}\right.$ $\left.<10^{14} / \mathrm{cm}^{3}\right)$ and high pressures $(P>10$ Torr) where Langmuir probe and optical emission diagnostics including the Stark effect are not accurate for density measurements. (C) 2003 American Institute of Physics. [DOI: 10.1063/1.1533104]

\section{INTRODUCTION}

Millimeter (mm) wave interferometry ${ }^{1,2}$ techniques along with polarimetry ${ }^{3,4}$ and optical emission diagnostics ${ }^{5,6}$ have been widely used as nonperturbing methods for plasma diagnostics. Faraday rotation-based polarimetry originally developed for magnetic field measurement can be used as an accurate densitometer for magnetized plasmas. However, the application of such polarimetry is limited to high-density magnetized plasmas at low gas pressure where electronneutral and electron-ion collision frequencies are small. At high gas pressure and collisionality, optical diagnostics including the Stark effect ${ }^{5}$ are used for plasma density and temperature characterizations. But the minimum plasma density requirement for these optical diagnostics to be accurate is very high $\left(n_{e} \geqslant 10^{14-15} / \mathrm{cm}^{3}\right){ }^{5,6}$ Interferometry provides a viable diagnostic tool to measure the average plasma density accurately over wide plasma density and gas pressure ranges.

Interferometry is primarily a technique to measure the refractive properties of the plasma which in turn depends on the plasma properties including the plasma density and collision frequency. The interferometer works on the MachZehnder principle $^{1}$ in which the plasma is in one arm of the two-beam interferometer. Let us consider the $\mathrm{mm}$ wave propagation in an infinite, uniform Lorentz plasma. At low collisionalities, the phase difference between the two arms with the plasma present to that without the plasma is a measure of the plasma density. ${ }^{1}$ In low pressure discharges where the wave frequency $(\omega)$ is always much greater than the plasma $\left(\omega_{p}\right)$ and collision frequency $\left(\nu_{\text {eff }}\right)\left(\omega \gg \omega_{p} \gg \nu_{\text {eff }}\right)$, the $\mathrm{mm}$ wave suffers almost little or no attenuation as it travels through the plasma. Therefore, only phase change

${ }^{a)}$ Electronic mail: kamran@cae.wisc.edu data are needed for a density measurement. In this case, a linear relationship exists between the line-average plasma density and the phase shift for a radially uniform plasma column. ${ }^{2}$ Also if $\omega_{p} \gtrsim \omega$ in low collisionality plasmas, the ordinary wave mode (o-mode) is in cutoff and interferometry data can not be obtained. On the other hand for high pressure discharges, where the collision frequency is higher than both the plasma and the wave frequency ( $\nu_{\text {eff }} \geqslant \omega \sim \omega_{p}$ ), we experimentally observe o-mode transmission for $\omega_{p} \geqslant \omega$ as predicted by the theory. The average plasma density for such highly collisional plasmas is proportional to the square root of the phase shift and is linearly related to the collision frequency. ${ }^{7}$ In this limiting case, an approximate refractive index is obtained for Lorentz plasma and the effect of collision frequency is incorporated in the formulation only through the phase function. This stronger dependence of the plasma density on collision frequency and reduced dependence on the phase shift introduce considerable error in the plasma density measurement at an intermediate pressure range ( 100 Torr $<P<300$ Torr) where the collision frequency is of the order of the wave frequency.

In a previous experiment carried out by our group, a 105 $\mathrm{GHz}$ interferometer was used to measure the average plasma density of a laser-initiated and rf sustained plasma. ${ }^{8}$ Using the collisionless approximation, an average plasma density of $3 \times 10^{12} \mathrm{~cm}^{-3}$ was measured for an argon plasma at 150 Torr with $2.5 \mathrm{~kW}$ of coupled rf power. The corresponding plasma phase change was $70^{\circ}$ over a $5 \mathrm{~cm}$ diameter chamber. In such high-pressure discharges, the dominant collision mechanism is the electron-neutral collision for which collision rates are compiled by Vahedi. ${ }^{9}$ This can be used to calculate the electron-neutral collision frequency as ${ }^{10}$ 


$$
\nu=n_{g} K \approx 3 \times 10^{9} \times \mathrm{P} \text { (Torr). }
$$

Here, $n_{g}$ is the neutral particle density $\left(\mathrm{m}^{-3}\right), K$ is the collision rate constant $\left(\mathrm{m}^{3} / \mathrm{s}\right)$, and $P$ is the neutral gas pressure in Torr. The corresponding electron-neutral collision frequency at 150 Torr is $1.5 \times 10^{11} \mathrm{~s}^{-1}$. If the effect of collision frequency in the highly collisional limit is considered as in Ref. 7 and discussed in more detail in Sec. III B, the predicted average plasma density increases by an order of magnitude to $2.8 \times 10^{13} \mathrm{~cm}^{-3}$. In this pressure and density regime where $\nu_{\text {eff }} \sim \omega \geqslant \omega_{p}$, neither the collisionless nor the high-collisional limits are valid and the predicted plasma density is not accurate.

At high gas pressures, the $\mathrm{mm}$ wave propagating through the plasma arm undergoes a phase change as well as strong attenuation. The wave attenuation is caused by the presence of high collisionality. In this situation, the plasma density has a complex dependence on phase change as well as on amplitude change and, therefore, the plasma density estimate using only phase change data is inaccurate. Therefore, a correct evaluation of plasma density can only be obtained if both phase change and amplitude change data are used.

In this article, we present a measurement and analysis technique where both amplitude change and phase change data are used simultaneously to uniquely determine both plasma density and effective collision frequency. The treatment does not limit the application of interferometry to the relative values of collision frequency and, hence, can be used for measurements at both low gas pressure $\left(\omega \gg \omega_{p} \gg \nu_{\text {eff }}\right)$ and high gas pressure $\left(\nu_{\text {eff }} \geqslant \omega, \omega_{p}\right)$. The analysis does not assume, $a b$ initio, a particular value of the collision frequency, rather than it calculates the collision frequency along with density using the phase and amplitude change data. In addition, insight in to the dominant loss mechanism can also be obtained from the temporal plasma decay plot. The experimental arrangement is presented in Sec. II, with the theoretical analysis presented in Sec. III, and results are discussed in Sec. IV.

\section{EXPERIMENTAL SETUP}

A $105 \mathrm{GHz}$ quadrature $\mathrm{mm}$ wave interferometry system (QBY-1A10UW, Quinstar Technology) is used to measure the plasma density and the effective collision frequency of a rf produced plasma. The rf source is a $10 \mathrm{~kW}$ solid-state unit (CX10K-S, Comdel, Inc.) with variable duty cycle $(90 \%-$ $10 \%)$, variable pulse repetition frequency $(100 \mathrm{~Hz}-1 \mathrm{kHz})$ and very fast $(\mu \mathrm{s})$ turn-on/off time. This fast turn-on/off time is needed to determine the dominant loss mechanisms as the plasma decays when the rf power is turned off. The rf power is coupled through a helical antenna that excites the $m=0$ transverse electric mode very efficiently using a capacitive matching network. The helical antenna is a five-turn coil of quarter-inch copper tube wound tightly over the $5 \mathrm{~cm}$ diameter pyrex plasma chamber. The coil is $10.0 \mathrm{~cm}$ long axially and has a $6 \mathrm{~cm}$ internal diameter. Figure 1 shows the schematic of the experimental system.

The interferometer works by using an in-phase and quadrature phase mixer to determine the phase and amplitude change of the $105 \mathrm{GHz} \mathrm{mm}$ wave signal going through

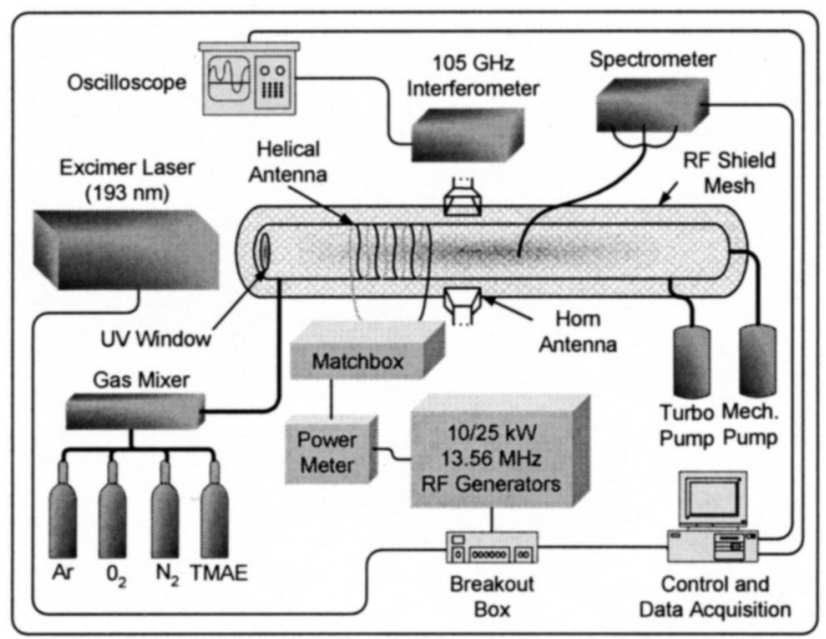

FIG. 1. Schematic of the laser-initiated and rf sustained plasma experiment.

the plasma. The data acquisition set up is shown schematically in Fig. 2. The two outputs are transferred to the computer through an oscilloscope with a general purpose interface bus and stored using a LABVIEW program. As the Gunn source and detector diodes are extremely sensitive to the rf radiation, the entire interferometer assembly is enclosed in a conducting box with slots cut in the box for the waveguides. In addition, cables with very high shielding $(\geqslant 90 \mathrm{~dB}$, Times Microwave Systems) have been used to reduce the noise level on the interferometer signal. The interferometric trace shown in the Fig. 3 is a function of time as the argon plasma decays when the rf power is turned off. A numerical program utilizing MATLAB solves for both phase and attenuation data to obtain the plasma density as well as the effective collision frequency. The density plot versus time also reveals the loss mechanisms including effective recombination coefficients at higher gas pressures.

\section{THEORY}

The propagation of electromagnetic waves in a plasma is determined by the value of the dielectric function. We assume a cold plasma approximation model since for most of

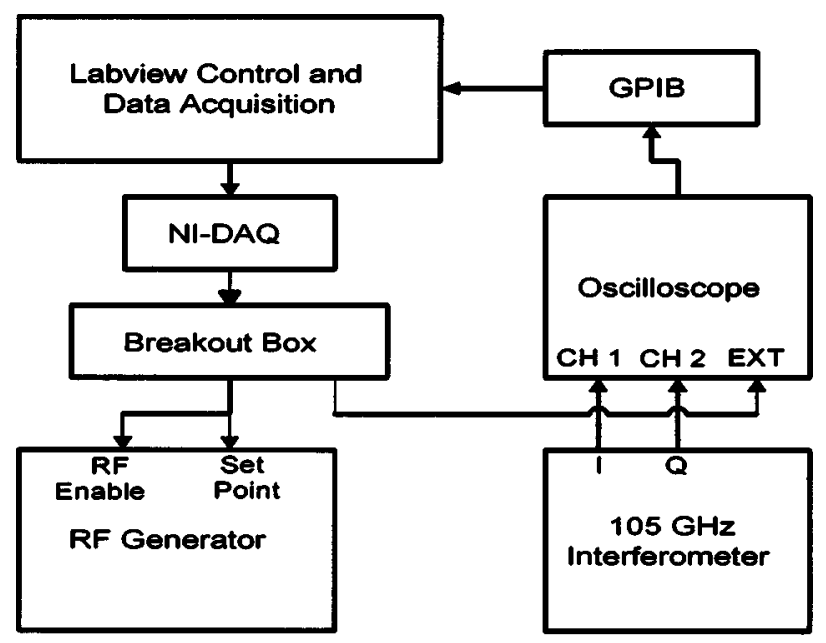

FIG. 2. Interferometer and data acquisition setup. 


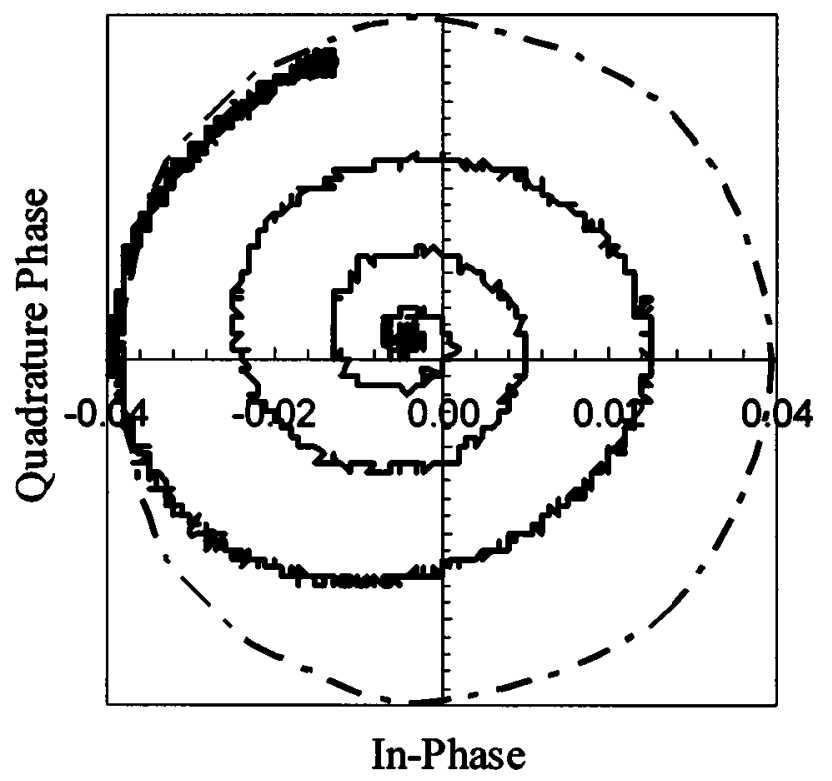

FIG. 3. Interferometer trace showing a nearly cutoff density of 9 $\times 10^{13} \mathrm{~cm}^{-3}$ in 10 Torr argon plasma at $1.0 \mathrm{~kW}$ using a five-turn helical antenna. The vacuum circle is represented by the dotted line. Measurements are made $10 \mathrm{~cm}$ away from the antenna.

the applications, the wave phase velocity is much greater than the electron thermal velocities and we can ignore thermal particle motion. Electron interaction is only through collective space-charge forces and the ions are assumed to form a stationary background.

The equation of motion for plasma electrons in the absence of a magnetic field is written as

$$
m \ddot{\mathbf{r}}=-e \mathbf{E}-\nu_{\mathrm{eff}} m \dot{\mathbf{r}},
$$

where $\mathbf{r}$ is the electron displacement vector and $\mathbf{E}$ is the electromagnetic field. We also define $\nu_{\text {eff }}$, the effective collision frequency for momentum transfer. If the electric field varies as $\exp (j \omega t)$, the displacement vector $\mathbf{r}$ is given as

$$
\mathbf{r}=\frac{e \mathbf{E}}{m \omega\left(\omega-j \nu_{\mathrm{eff}}\right)} ; \dot{\mathbf{r}}=\frac{-e \mathbf{E}}{m} \frac{\left(\nu_{\mathrm{eff}}-j \omega\right)}{\left(\omega^{2}+\nu_{\mathrm{eff}}^{2}\right)} .
$$

The complex conductivity $\sigma$ is given as

$$
\sigma \equiv \sigma_{r}+j \sigma_{i}=-\frac{n_{e} e \dot{r}}{E}=\frac{n_{e} e^{2}}{m} \frac{\left(\nu_{\mathrm{eff}}-j \omega\right)}{\left(\omega^{2}+\nu_{\mathrm{eff}}^{2}\right)},
$$

and the complex relative dielectric constant for a linear medium is given by

$$
\frac{\varepsilon}{\varepsilon_{0}}=\kappa=\kappa_{r}+j \kappa_{i}=1-j \frac{\sigma}{\varepsilon_{0} \omega}=1-\frac{\omega_{p}^{2}}{\omega^{2}+\nu_{\mathrm{eff}}^{2}}\left(1+j \frac{\nu_{\mathrm{eff}}}{\omega}\right),
$$

while $\omega_{p}$ is the plasma frequency and $\varepsilon_{0}$ is the free space permittivity. The complex refractive index and the complex propagation constants are

$$
n=\frac{c}{v}=\mu_{r}-j \chi=\kappa^{1 / 2} ; \quad \gamma=\alpha+j \beta=\frac{\omega}{c}(j \mu)=\frac{\omega}{c} \sqrt{\kappa} .
$$

Here $\alpha=\chi \omega / c$ is the attenuation constant in $\mathrm{Np} / \mathrm{m}$ and $\beta$ $=\mu_{r} \omega / c$ is the phase constant in $\mathrm{rad} / \mathrm{m}$. The solution for the plane wave phase and attenuation constants yields

$$
\begin{aligned}
\beta_{p}= & \frac{\omega}{c}\left\{\frac{1}{2}\left(1-\frac{\omega_{p}^{2}}{\omega^{2}+\nu_{\mathrm{eff}}^{2}}\right)+\frac{1}{2}\left[\left(1-\frac{\omega_{p}^{2}}{\omega^{2}+\nu_{\mathrm{eff}}^{2}}\right)^{2}\right.\right. \\
& \left.\left.+\left(\frac{\omega_{p}^{2}}{\omega^{2}+\nu_{\mathrm{eff}}^{2}} \frac{\nu_{\mathrm{eff}}}{\omega}\right)^{2}\right]^{1 / 2}\right\}^{1 / 2}, \\
\alpha_{p}= & \frac{\omega}{c}\left\{-\frac{1}{2}\left(1-\frac{\omega_{p}^{2}}{\omega^{2}+\nu_{\mathrm{eff}}^{2}}\right)+\frac{1}{2}\left[\left(1-\frac{\omega_{p}^{2}}{\omega^{2}+\nu_{\mathrm{eff}}^{2}}\right)^{2}\right.\right. \\
& \left.\left.+\left(\frac{\omega_{p}^{2}}{\omega^{2}+\nu_{\mathrm{eff}}^{2}} \frac{\nu_{\mathrm{eff}}}{\omega}\right)^{2}\right]^{1 / 2}\right\}^{1 / 2} .
\end{aligned}
$$

Assuming a plasma slab of uniform average density profile, the total change in phase and amplitude for interferometric signal are given as

$$
\Delta \varphi=\int_{0}^{d}\left(\beta_{0}-\beta_{p}\right) d r ; \quad \Delta A=\int_{0}^{d}\left(\alpha_{0}-\alpha_{p}\right) d r .
$$

Here $\beta_{0}$ and $\alpha_{0}$ are the free space values and $\beta_{p}$ and $\alpha_{p}$ are the plasma values. A numerical program in MATLAB is used to solve for plasma density and $\nu_{\text {eff }}$ simultaneously from experimentally measured $\Delta \phi$ and $\Delta A$ values.

\section{A. Low collision frequency limit}

In the approximation of low collision frequencies $(\omega$ $\left.\gg \omega_{p} \gg \nu_{\text {eff }}\right)$ where most interferometry operates, the phase constant and attenuation constant are given as

$$
\begin{aligned}
& \beta_{p}=\frac{\omega}{c}\left(1-\frac{\omega_{p}^{2}}{\omega^{2}}\right)^{1 / 2} \approx \frac{\omega}{c}\left(1-\frac{\omega_{p}^{2}}{2 \omega^{2}}\right) ; \\
& \alpha_{p}=\frac{\nu_{\mathrm{eff}} \omega_{p}^{2}}{2 \omega^{2} c}\left(1-\frac{\omega_{p}^{2}}{\omega^{2}}\right)^{-1 / 2} .
\end{aligned}
$$

In the limit of $\omega>\omega_{p}, \alpha_{p}$ is small and, therefore, the wave suffers almost no attenuation. ${ }^{2}$ Hence, the plasma density can be expressed in this limit for uniform density profile using Eq. (7) as

$$
\begin{aligned}
n_{e}=\left(\frac{4 \pi c \varepsilon_{0} m_{e}}{e^{2}}\right) \frac{f \Delta \phi}{d} & =1.187 \times 10^{6} \frac{f \Delta \phi}{d} \\
& =2.073 \frac{f \Delta \phi}{d} \mathrm{~cm}^{-3} .
\end{aligned}
$$

Here, the phase change is in degrees, the diameter in centimeters, and wave frequency is in $\mathrm{s}^{-1}$.

\section{B. High collision frequency limit}

For highly collisional plasmas at high gas pressures $\left(\nu_{\text {eff }} \gg \omega \geqslant \omega_{p}\right)$, the phase constant is written as

$$
\beta_{p}=\frac{\omega}{c}\left\{\frac{1}{2}+\frac{1}{2}\left[1+\frac{\omega_{p}^{4}}{\omega^{2} \nu_{\mathrm{eff}}^{2}}\right]^{1 / 2}\right\}^{1 / 2} \approx \frac{\omega}{c}\left[1+\frac{\omega_{p}^{4}}{8 \omega^{2} \nu_{\mathrm{eff}}^{2}}\right] .
$$

For a radially uniform plasma profile, 
TABLE I. Comparison of plasma density computation using $105 \mathrm{GHz}$ interferometer for $5 \mathrm{~cm}$ diameter tube.

\begin{tabular}{|c|c|c|c|c|c|}
\hline $\begin{array}{c}\Delta \Phi \\
\text { (degrees) }\end{array}$ & $\begin{array}{l}\text { Attenuation } \\
\quad(\mathrm{dB})\end{array}$ & $\begin{array}{l}\nu_{\text {eff }} \\
\left(\mathrm{s}^{-1}\right)\end{array}$ & $\begin{array}{c}n_{e}\left(\mathrm{~cm}^{-3}\right) \\
\text { Using both phase } \\
\text { and amplitude data } \\
{[\text { Eq. (9)] }}\end{array}$ & $\begin{array}{c}n_{e}\left(\mathrm{~cm}^{-3}\right) \\
\text { Collisionless limit } \\
{[\text { Eq. (11) }]^{\mathrm{a}}}\end{array}$ & $\begin{array}{l}n_{e}\left(\mathrm{~cm}^{-3}\right) \\
\text { Highly collisional } \\
\text { plasma }[\text { Eq. (13) }]^{\mathrm{b}}\end{array}$ \\
\hline 100.0 & 0.023 & $1.0 \times 10^{9}$ & $4.30 \times 10^{12}$ & $4.32 \times 10^{12}$ & $7.38 \times 10^{10}$ \\
\hline 479.6 & 6.51 & $5.7 \times 10^{10}$ & $2.01 \times 10^{13}$ & $2.07 \times 10^{13}$ & $9.22 \times 10^{12}$ \\
\hline 175.0 & 4.07 & $1.0 \times 10^{11}$ & $7.65 \times 10^{12}$ & $7.57 \times 10^{12}$ & $9.77 \times 10^{12}$ \\
\hline 70.0 & 8.60 & $5.3 \times 10^{11}$ & $4.99 \times 10^{12}$ & $3.0 \times 10^{12}$ & $3.27 \times 10^{13}$ \\
\hline 30.0 & 6.93 & $1.0 \times 10^{12}$ & $4.30 \times 10^{12}$ & $1.29 \times 10^{12}$ & $4.04 \times 10^{13}$ \\
\hline 100.0 & 23.65 & $1.0 \times 10^{12}$ & $1.45 \times 10^{13}$ & $4.32 \times 10^{12}$ & $7.38 \times 10^{13}$ \\
\hline 150.0 & 56.1 & $1.5 \times 10^{12}$ & $4.25 \times 10^{13}$ & $6.49 \times 10^{12}$ & $1.35 \times 10^{14}$ \\
\hline
\end{tabular}

${ }^{\mathrm{a}}$ See Ref. 2.

${ }^{\mathrm{b}}$ See Ref. 7.

$$
n_{e}=38.6 \nu_{\mathrm{eff}}\left(\frac{f \Delta \phi}{d}\right)^{1 / 2}=5.09 \times 10^{-5} \nu_{\mathrm{eff}}\left(\frac{f \Delta \phi}{d}\right)^{1 / 2} \mathrm{~cm}^{-3} .
$$

Here again, frequencies are in $\mathrm{s}^{-1}$, the diameter is in centimeter, and phase changes are in degrees. ${ }^{7}$

\section{Determination of recombination coefficients}

A measurement of the dominant loss mechanism in a plasma can be obtained from the temporal plot of plasma density decay. Each point on the trace in Fig. 3 corresponds to the instantaneous phase and amplitude change of the $\mathrm{mm}$ wave signal traveling through the plasma. The instantaneous plasma density and corresponding effective collision frequency are obtained by simultaneously solving for the instantaneous phase and amplitude change data and a plot of the plasma density and effective collision frequency as a function of time is obtained. From the density decay plot, an estimate of the effective recombination rate for the plasma at higher gas pressures is obtained using the continuity equation.

The continuity equation, including the ambipolar diffusion term, the ionization frequency term, $\nu_{i z}$, and two- and three-body recombination terms, is given by

$$
\partial n_{e} / \partial t=D_{n} \nabla^{2} n_{e}+\nu_{i z} n_{e}-\alpha_{r} n_{e}^{2}-\beta_{r} n_{g} n_{e}^{2}
$$

Here, $\alpha_{r}, \beta_{r}$, and $n_{g}$ are the two-body recombination rate, three-body recombination rate, and the neutral gas pressure, respectively. For a temporally decaying plasma without any ionizing source, the continuity equation takes the form

$$
\partial n_{e} / \partial t=D_{n} \nabla^{2} n_{e}-\alpha_{r} n_{e}^{2}-\beta_{r} n_{g} n_{e}^{2} .
$$

In low to moderate pressure discharges, plasma diffusion to the chamber is the principal loss mechanism for plasma decay. However, at higher gas pressures ( $P>100$ mTorr), two-body recombination starts to dominate the plasma decay characteristics and limits the peak plasma density obtainable at high pressures. The number of particles lost due to twobody recombination in argon above 100 mTorr is much higher than the number lost due to diffusion. ${ }^{8}$ With a further increase in pressure, three-body recombination processes involving an ion, an electron, and a neutral, contributes to the plasma decay and becomes the dominant loss mechanism at very high pressures. An estimate of the gas pressure, where the three-body effect in argon becomes comparable to the two-body effect, can be obtained. For argon gas, the twobody recombination coefficient ${ }^{11} \alpha_{r}^{\mathrm{Ar}}=6.5 \times 10^{-8} \mathrm{~cm}^{3} \mathrm{~s}^{-1}$ and three-body recombination coefficient (Ref. 12) $\beta_{r}^{\mathrm{Ar}}$ $=10^{-26} \mathrm{~cm}^{6} \mathrm{~s}^{-1}$. Using $\alpha_{r}^{\mathrm{Ar}}=\beta_{r}^{\mathrm{Ar}} n_{g}$, the argon pressure at which these two effects become comparable is 202 Torr. However, the pressure range where the contribution to plasma decay from three-body recombination is important in the calculation differs widely for different gases. In some cases, it is more appropriate to define an effective recombination coefficient, $\alpha_{\text {eff }}$, which includes two- and three-body recombination effects, and can be easily determined from the plasma density decay plot. For the pressure range of interest, we have

$$
\partial n_{e} / \partial t=-\left(\alpha_{r}+\beta_{r} n_{g}\right) n_{e}^{2}=-\alpha_{\mathrm{eff}} n_{e}^{2} .
$$

Here $\alpha_{\text {eff }}$ is the effective recombination term that includes two- and three-body recombination effects.

\section{RESULTS AND DISCUSSION}

The results for the plasma density computation using Eqs. (9), (11), and (13) are presented in Table I, for typical phase change and collision frequency data in a rf produced plasma source at high gas pressures. From the experimentally determined phase and attenuation data, the plasma density and effective collision frequency are determined using the analysis presented herein. The collision frequency and phase change data are then used to calculate the plasma density using Eqs. (11) and (13). Plasma density and effective collision frequency have a complex dependence on phase change and attenuation data. At low gas pressure $(\sim 1$ Torr argon), where the collision frequency is low (1.0 $\times 10^{9} \mathrm{~s}^{-1}$ ), the plasma density estimate using the collisionless approximation is the same as that obtained by the present method. However, the plasma density estimated using the highly collisional plasma approximation is two orders of magnitudes lower. When the pressure is raised to 300 Torr and the rf power is also raised to obtain a similar phase shift of $100^{\circ}$ as obtained for gas pressure for 1 Torr, the effective collision frequency is $1.0 \times 10^{12} \mathrm{~s}^{-1}$. The plasma density computed using the collisionless approximation remains the same, since it is assumed to be independent of the collision frequency. However, the predicted density increases from $7.38 \times 10^{10} \mathrm{~cm}^{-3}$ at low $\nu_{\text {eff }}$ to $7.38 \times 10^{13} \mathrm{~cm}^{-3}$ when computed using the highly collisional plasma approximation in 

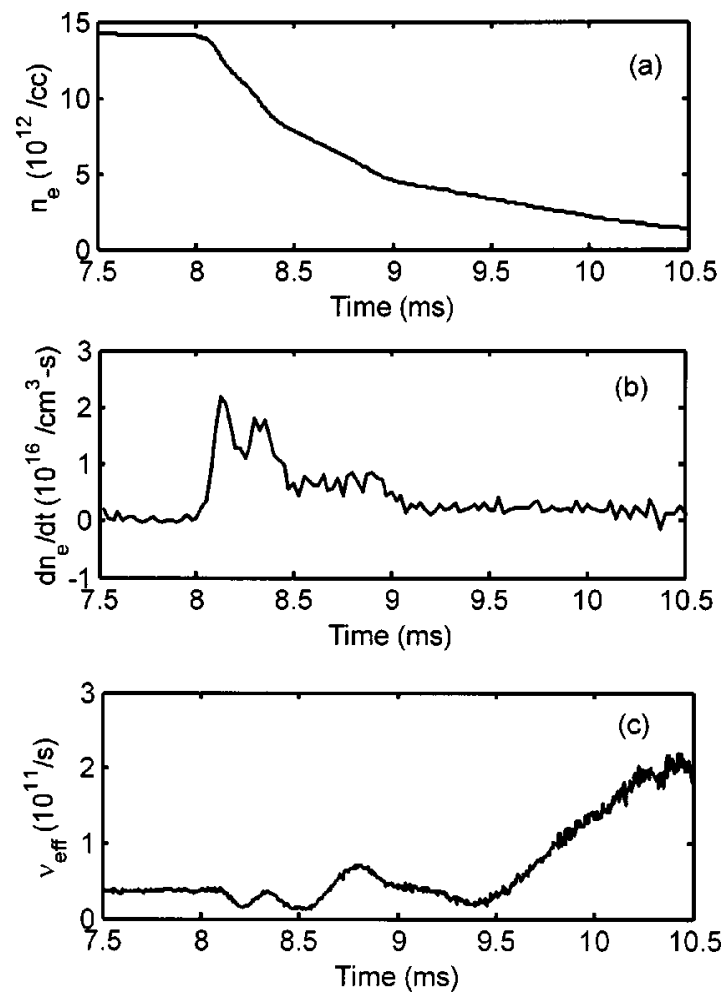

FIG. 4. For a temporally decaying plasma, MATLAB plots of (a) plasma density, (b) density decay, and (c) effective collision frequency as a function of time obtained from $\mathrm{mm}$ waves amplitude and phase shift data.

Eq. (13). The plasma density when computed using the more accurate phase change and attenuation data changes only by an order of magnitude from $4.30 \times 10^{12}$ to 4.77 $\times 10^{13} \mathrm{~cm}^{-3}$. It is clear from this analysis that density measurements in the presence of high collisionality at intermediate pressures where the collision frequency is less than the wave frequency must include the attenuation data, otherwise, it will lead to significant error. Since both the phase change and attenuation data are used in the present method, it is independent of the collision frequency range.

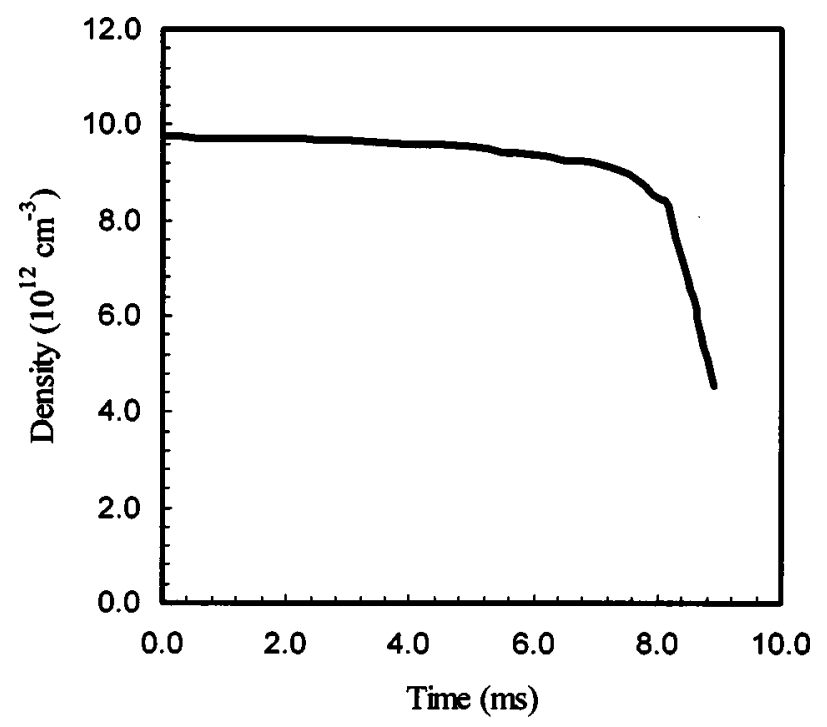

FIG. 5. Density decay plot as a function of time for a temporally decaying argon plasma. For a 50 Torr argon discharge at $3.0 \mathrm{~kW}$ coupled rf power and flow rate of $20 \mathrm{slm}$, the effective recombination coefficient is $\alpha_{\text {eff }}=2.0$ $\times 10^{-10} \mathrm{~cm}^{3} / \mathrm{s}$.

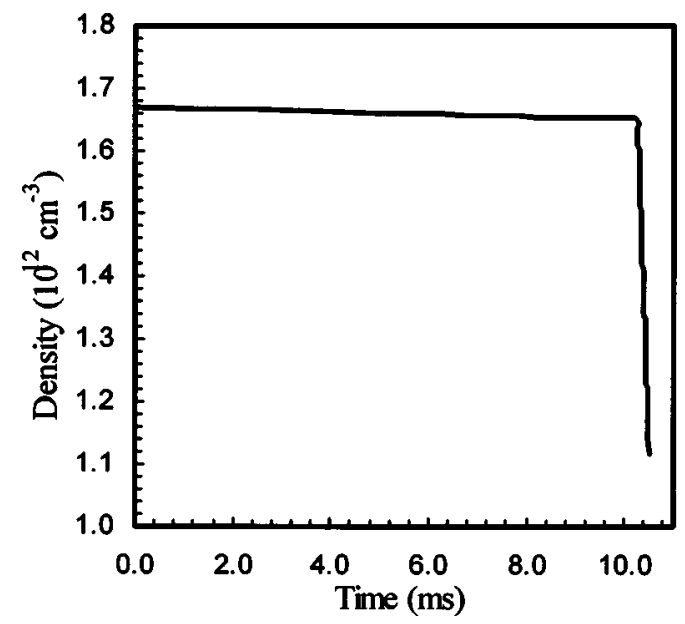

FIG. 6. The plasma density decay plot for the static 50 Torr argon discharge at $3.0 \mathrm{~kW}$. The corresponding recombination coefficient for this case is $\alpha_{\text {eff }}=1.0 \times 10^{-8} \mathrm{~cm}^{3} / \mathrm{s}$.

The characteristics of plasma decay and the nature of the loss mechanism can only be investigated if the rf generator turn-on/off time is faster than the plasma decay time. A solidstate rf generator with the fast turn-on/off time is suitable for these measurements. From the interferometer trace shown in Fig. 3, the average plasma density and effective collision frequency are calculated for a temporally decaying plasma. The density decay plot is obtained by numerical differentiation of the density plot. Using Eq. (16), the recombination coefficient is obtained. An interactive graphical program in MATLAB has been developed to analyze the interferometer data and a typical plot is shown in Fig. 4. In an experiment to investigate the effect of gas flow on rf produced plasmas, the gas flow rates were adjusted using a mass flow meter. Two test cases of 50 Torr argon plasmas are presented here to explain the determination of recombination coefficients. In the static case, or zero-flow rate case, the evacuated chamber is raised to 50 Torr by opening the gas inlet valve and closing the pump valve. In the flow case, a mechanical pump is kept running with the flow rates adjusted to 20 standard liters per minute (slm) using a mass flow meter at 50 Torr. A delay of 5-20 ms can be introduced between the oscilloscope trigger and rf power turn off. As shown in Figs. 5 and 6, the plasma density remains constant initially for $5-7 \mathrm{~ms}$ (delay) and then it decays sharply. A higher density decay rate and recombination coefficient $\left(1.0 \times 10^{-8} \mathrm{~cm}^{3} / \mathrm{s}\right)$ are obtained for the static case. The recombination rate is much lower (2.0 $\times 10^{-10} \mathrm{~cm}^{3} / \mathrm{s}$ ) when gas flow is maintained at $20 \mathrm{slm}$.

This diagnostic is particularly valuable for collisional plasmas of moderate densities $\left(n_{e}<10^{14} / \mathrm{cm}^{3}\right)$ at higher gas pressures, where probe and optical emission diagnostics are not suitable for density measurements. We are currently utilizing this diagnostic for characterization of mixed flow airargon, laser-initiated, rf-sustained collisional plasmas.

\section{ACKNOWLEDGMENTS}

This research was supported by Air Force Office of Scientific Research Grant No. F49620-00-1-0181 and in part by NSF Grant No. ECS-9905948. 
${ }^{1}$ I. H. Hutchinson, Principles of Plasma Diagnostics (Cambridge University Press, Cambridge, UK, 1987), p. 87.

${ }^{2}$ C. B. Wharton, in Plasma Diagnostic Techniques, edited by R. H. Huddlestone and S. L. Leonard (Academic, New York, 1965), p. 477.

${ }^{3}$ F. C. Jobes and D. K. Mansfield, Rev. Sci. Instrum. 63, 5154 (1992).

${ }^{4}$ T. Akiyama, E. Sato, T. Nozawa, S. Tsuji-Iio, R. Shimada, H. Murayama, K. Nakayama, S. Okajima, K. Tanaka, K. Watanabe, T. Tokuzawa, and K. Kawahata, Rev. Sci. Instrum. 72, 1073 (2001).

${ }^{5}$ H. R. Griem, Principles of Plasma Spectroscopy (Cambridge University Press, Cambridge, UK, 1997), p. 258.
${ }^{6}$ W. Lochte-Holtgreven in Plasma Diagnostics, edited by W. LochteHoltgreven (North-Holland, Amsterdam, 1968), p. 186.

${ }^{7}$ M. Laroussi, Int. J. Infrared Millim. Waves 20, 1501 (1999).

${ }^{8}$ K. L. Kelly, J. E. Scharer, E. S. Paller, and G. Ding, J. Appl. Phys. 92, 698 (2002).

${ }^{9}$ V. Vahedi, R. A. Stewart, and M. A. Lieberman, J. Vac. Sci. Technol. A 11, 1275 (1993).

${ }^{10}$ M. A. Lieberman and A. J. Lichtenberg, Principles of Plasma Discharges and Materials Processing (Wiley, New York, 1994), p. 80.

${ }^{11}$ M. Wojcik and M. Tachiya, J. Chem. Phys. 110, 10016 (1999).

${ }^{12}$ M. J. Seaton, Mon. Not. R. Astron. Soc. 119, 81 (1959). 